Original Article - Clinical Science

\title{
Study methodology and diabetes control in patients from the non-English Diabetes Management Project (NEDMP)
}

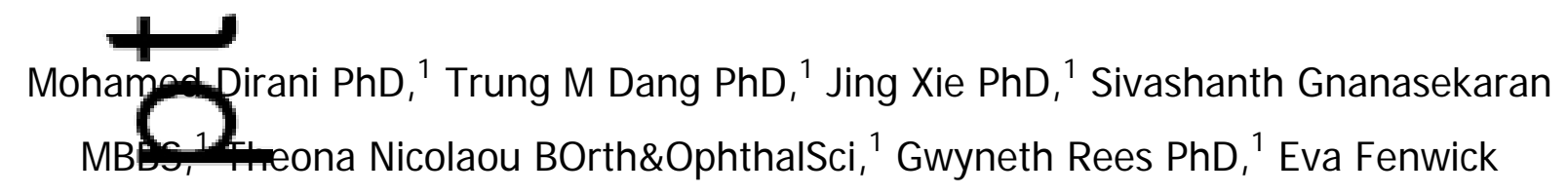

- $\mathrm{PhD}^{1,2,4}$ and Ecosse E Lamoureux $\mathrm{PhD}^{1,2,3,4}$

1. Centre for Eye Research Australia, University of Melbourne, Royal Victorian (E) e Ear Hospital, East Melbourne, Australia

2. Singapore Eye Research Institute, Singapore National Eye Centre, Singapore

3. Eepartment of Ophthalmology, National University of Singapore and National University Health System, Singapore

4. Duke-NUS Medical School, Singapore రে

Correspondence: Dr Mohamed Dirani, Centre for Eye Research Australia, Royal

Victe:ran Eye and Ear Hospital, 32 Gisborne Street, East Melbourne, Victoria,

Australia 3002

Email:dirani@unimelb.edu.au

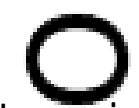

Short running title: The non-English Diabetes Management Project

Receiva 5April 2016; accepted 19 J uly 2016

Confiucoifinterest: None

Funding sojurces: This study was supported under the Australian Research

Council's Linkage Grant funding scheme (LP100200662). MD, GR and EF are

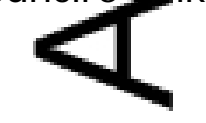

This is the author manuscript accepted for publication and has undergone full peer review but has not been through the copyediting, typesetting, pagination and proofreading process, which may lead to differences between this version and the Version of Record. Please cite this article as doi: 10.1111/ceo.12807

This article is protected by copyright. All rights reserved. 
supported by National Health and Medical Research Council Fellowships (\#1090466, \#1061801 and \#1072987). CERA receives Operational Infrastructure Support from the Victorian Government.

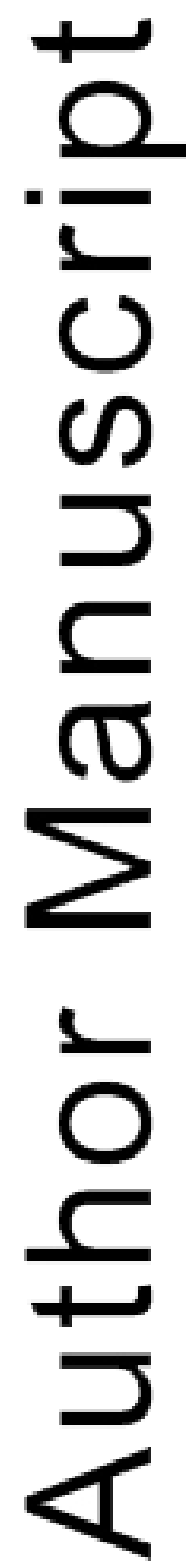

This article is protected by copyright. All rights reserved. 


\section{ABSTRACT}

Background: To describe the clinical characteristics of non-English speaking patients from the Diabetes Management Project (NEDMP), and compare their diabetes management and severity of diabetic retinopathy (DR) with the Englishspeaking DMP sample (EDMP).

Desigh: A prospective study was conducted on non-English speaking adults with diabeterwo attended the Royal Victorian Eye and Ear Hospital.

Participents: 136 (90.1\%) non-English speaking adults were assessed, with a mean Ge 72.2 years (range: 50-88 years); 74 (54.4\%) were male.

MethodpParticipants completed interviewer-administered questionnaires and underwent visual acuity, fundus photography, optical coherence tomography, biochemisty and anthropometric measurements. The EDMP assessed 609 patients in 200 wring a similar protocol.

Main Uurcome Measures: Type and duration of diabetes, diabetes control and diabet Cefnopathy.

Resultal of 127 (93.4\%) and 8 (5.9\%) participants reported having type 2 and typ 2 diabetes, respectively, with a median (IQR) duration of 17 (14) years. The proportion of patients with poor diabetes control (HbA1c $\geq 7 \%$ ) in the NEDMP was similar to the EDMP ( $64.0 \%$ and $68.2 \%$, respectively; $p=0.411)$. A significantly higher praportion of patients with DR in the NEDMP were found to have poor diabetentrol (HbA1c $\geq 7 \%$ ) compared to those without DR ( $80.9 \%$ vs. $50.0 \%$, $\mathrm{p}=0.0$ (3). Almost two-thirds of NEDMP patients (74/118) had DR and 23\% (27/115) had diabetic macular edema. The prevalence of DR was similar between the NEDMP and EDARstudies, ranging from $25-30 \%$ and $28-29 \%$.

Conclusions: The clinical characteristics, diabetes control, and DR severity of Englin nd non-English-speaking patients were similar. The high proportion of poor diabetes management in non-English speaking patients with DR suggests 
educational and behavioural interventions to improve glycaemic control are warranted.

Keywords: Ophthalmology publication, Diabetes, non-English, Diabetic retinopathy I NTRODUCTI ON

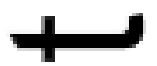

Diabetes mellitus (DM) is a chronic medical condition that affects approximately 1.2 millien-tralians, with a further 500,000 undiagnosed cases [1, 2]. It has been the prevalence of DM will almost double in Australia by 2025, from $7.4 \%$ ( 11) $4 \%$, posing major public health and economic challenges [1, 2]. One of the migropscular complications associated with DM, is diabetic retinopathy (DR) which is the most common and leading cause of blindness in working aged adults in most devel pped nations [3].

Optimanagement of risk factors such as blood glucose, lipids and blood pressure to reduce Tsk of DR is well known and supported by current Australian National Healtr addMedical Research Council (NHMRC) guidelines [4]. However, recently our grou English-speaking adults with type 1 and 2 DM (the Diabetes Management Project, or EDMP), and reported that $76.3 \%$ of adults with DR had poor glycemic control $\left(\mathrm{HbA}_{1 \mathrm{c}} \geq 7 \%\right)$ [5]. This finding indicates that patients are still not adkering to the management of their blood glucose and diabetes. The causes of inadequate diabetes control in Australia are multifactorial, such as socioeconomic status, aceduced accessibility to care and lack of education, lack of community aware ess, poor compliance to treatment and management regimes, and individuals' lack of knowledge about diabetes control $[5,6]$. These types of barriers limit an individuacity to make the behavioral changes required to reduce the risk of diabetes complications.

One timitations of the EDMP was its lack of generalizability, as approximately one third of people were excluded because they were non-English speaking. Access 
and understanding of healthcare information in both community and healthcare settings are critical to the management of diabetes, and individuals from non-English speaking backgrounds may be disadvantaged due to cultural and language barriers, as has been reported in the Asian-American population [7], Hispanic Americans [8] and Tamil speaking Indians in Singapore [9]. To date, there have been no Australian basea studies that have investigated the independent risk factors and barriers to optimd diabetes care in non-English speaking adults with and without DR. The we developed a parallel non-English speaking DMP study (the NEDMP), with simiter objectives and testing protocol to the EDMP, but using a different sampling a recruitment method to account for the logistical difficulties associated with research in non-English speaking populations. In this paper, we describe and compare the clinical characteristics of NEDMP, patients including severity of DR and level of diabetes control, with the EDMP sample.

\section{METHOOS \\ (U)}

Stud

Recruitment and data collection for this cross-sectional study took place from J anuary 2011 to November 2013. The inclusion criteria for the NEDMP were: 1) individuals with type 1 or 2 diabetes; 2) aged 18 years or older; 3) non-English speakipgindividuals who required Arabic, Cantonese, Italian, Greek, Mandarin or Vietna Moos interpreters; 4) living independently; and 5) adequate cognitive capaciy, as assessed using a validated six-item cognitive impairment test [10]. Individuals who failed the fifth criteria (score $>6$ ) were not excluded from participetion in the project; however their non-clinical data was removed from the main anarysis. The five languages were selected as they represented approximately two 5 of the non-English speaking patients at the Royal Victorian Eye and Ear Hospital (RVEEH). The inclusion criteria were determined during the screening phase 
of the study and then later confirmed face-to-face by trained interviewers and qualified interpreters at the point of recruitment in the corresponding eye clinics. Ethical approval was provided by the RVEEH Human Research and Ethics Committee $(08 / 815 \mathrm{H})$. Each participant provided written informed consent for their involvement in the study after being provided with information outlining the aims and method of the NEDIVIP, possible adverse outcomes, confidentiality policies and data storage procedured. The NEDMP protocol adhered to the tenets of the Declaration of Helsiniand all privacy requirements were met. The RVEEH and Diabetes Victoria were cortureorative partners involved in the NEDMP.

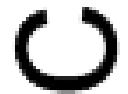

NEDMP sareening and recruitment

Participants were recruited from the general ophthalmology and specialised retinal clinics at the RVEEH. A day prior to the aforementioned clinics, trained staff screenospital patient information management services (PIMS) reports and flaggecraireligible individuals (those who had diabetes and required an interpreter) using (E) notification slip placed in the file. Demographic data, including the indivame, gender, date of birth, RVEEH patient identification number and contact details were collected for eligible patients. All medical records for a total of five clinical sessions were screened weekly. If inadequate information was available from the patient file, these files were flagged and then classified as eligible or ineligible the point of recruitment.

Traine ruiters, along with interpreters, were stationed at the corresponding clinic sites a orthoptists conducting the preliminary testing would alert recruiters to an eligible patient. Patients were then approached and invited to participate in the study. point, patients were categorised into one of four groups: 1) agreed to participate, 2) declined to participate; 3) would need further thought/information befo a reeing to participate; 4) missed by recruiter. Individuals who fell into the 
latter two categories were later invited to take part in the NEDMP via a telephone call by an interpreter.

Individuals who were undecided or who agreed to participate in the study were given language-specific recruitment packs containing a cover letter, a NEDMP brochure and flyer, a patient information sheet, a consent form, fasting instructions and a page to allow patients to record their current medications.

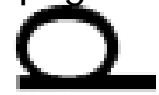

DMPapapointment times

An appoment time was scheduled for individuals who agreed to participate, typicaly wi)hin two weeks of the recruitment date. An appointment confirmation letter waprovided at recruitment or sent by mail. With the assistance of interpreters, a reminder telephone call was also made 2-4 days prior to the scheduled ppointment. Individuals were encouraged to be accompanied by a family memb day of the appointment. All participants were provided with a food and beverage voucher that could be used at the hospital's cafeteria.

ד

Testingocol

Each pantipant underwent a series of examinations, including a comprehensive eye assessment, biochemistry and anthropometric measurements, and a general questinnaire alongside a number of interviewer-administered behavioural questignires. All assessments were performed at the Centre for Eye Research Australiand in Melbourne, Australia, with testing duration varying from 2 to 4 hours or each participant.

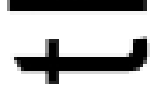

Eye Exominations

The NEUIMP examination procedure follows that used in the original EDMP study, which $s$ designed to ensure testing efficiency and data quality [3]. The NEDMP protocol was divided into four stages: (1) registration and consent, blood and urine 
collection, visual acuity (VA) assessment, dilation and breakfast; (2) general questionnaire administration and anthropometric measurements; (3) fundus photography, optical coherence tomography (OCT) scans, intraocular lens (IOL) Master, autorefraction and blood pressure (BP) measurements; and (4) completion of behavioural and psychosocial questionnaires. At all stages of the protocol, a researtener and accredited interpreter were present. Full details of the testing procedure have been published previously [5] and the following section outlines the protociefly, detailing any differences from the original study. Study interpreters were required to sign the appropriate documentation stating that they are qualified to trar(slat) speech and writing from the English language into the specified languagegf the participant.

\section{Blood and rine collection}

Blood samples were collected to determine glucose and lipid levels and albumin/creatinine ratio, respectively. A total of $34.5 \mathrm{~mL}$ of blood was collected from each aridpant via venipuncture into five separate tubes. This allowed for quarticination of low-density lipoprotein ( $L D L)$, high-density lipoprotein (HDL), triglycerietes, fasting glucose levels and glycosylated haemoglobin ( $\mathrm{HbA}_{1 \mathrm{c}}$ ) level. 18 $\mathrm{mL}$ was processed and stored for future genetic and molecular biology studies. A midstream urine sample $(50 \mathrm{~mL})$ was collected to determine albumin/creatinine ratio. Bipenemical parameters were analysed at Melbourne Pathology, Melbourne, Australind results delivered electronically through a secure interface (Webster online results services).

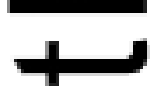

Visual y (VA) assessment

Refractive status was obtained from the participant's file prior to the clinical exareing ion. Distance monocular and binocular VA with current correction (termed 'presenting $V A^{\prime}$ ' hereafter) was assessed using a 3-metre logMAR chart (National 
Vision Research Institute, University of Melbourne, Australia). A logMAR word card was used to assess both monocular and binocular presenting near VA. Although this word card is calibrated for measurement at $25 \mathrm{~cm}$, the test was conducted at the participant's preferred reading distance. Near VA was recorded in both logMAR and $\mathrm{N}$, or by indicating that even the largest line was unable to be read.

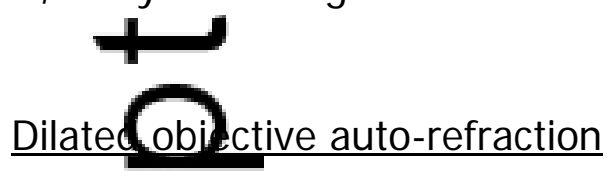

Participes had both eyes dilated using Tropicamide 1\% (Minims, Chauvin Pharmeterticals, Ltd., Surrey, UK). Approximately 25 minutes after instillation of the drops, (aut)-refraction assessment was conducted using the hand-held Retinomax 2 (Nikon Tokyo, Japan). A total of five readings were taken for each eye, and the average value was recorded.

IOL master

Cornear curvature (keratometry, $\mathrm{K} 1$ and $\mathrm{K} 2$ ), axial length (anteroposterior diameter) and ar teidr chamber depth of both eyes was obtained using the IOL master (Carl Zeis malitec, Oberkochen, Germany). To ensure consistency of results, at least three consecutive readings were taken for each ocular biometry measurement. Axial length and anterior chamber depth readings were all within $0.02 \mathrm{~mm}$, with a soundnoise atio of greater than 2.0 .

Fundushotography/diabetic and vascular grading

A non mydriatic retinal camera (Canon CR6 - 45NM, Canon Inc, Tokyo, Japan) was used tp take two field, $45^{\circ}$, digital, non-stereo, colour fundus photographs of both eyes each participant. Diabetic retinopathy grading using the 'Modified 2Standarurfeld Color Fundus Photography Procedure' (Retinal Vascular Imaging Cent c ading Protocol \#01 - Assessment of DR) was performed by the Retinal Vascular Imaging Centre, which is part of the Centre for Eye Research Australia. A 
semi-automated retinal vasculature imaging program, that quantitatively measures retinal vascular geometric parameters, was employed to grade retinal vasculature.

\section{Ocular Coherence Tomography (OCT)}

Retinal nerve fibre layer scans and fast macular scans with retinal map analysis (fast macutar thickness map, retinal thickness/volume tabular) were obtained using the OCT Sratus Model 3000 (Carl Zeiss Meditec Inc., Dublin, CA, USA).

Anthropontric and BP measurements A wall (mo)ted, adjustable measuring scale (Surgical and Medical products, Guangahry China) and a calibrated digital scientific weight scale (Oregon Scientific, Beijing, China) allowed measurement of height and weight, respectively. These values allo) ved determination of the participant's body mass index (BMI) using the univercall-recognised formula $\left(\mathrm{W}[\mathrm{kg}] / \mathrm{H}[\mathrm{m}]^{2}\right)$. BMl values allowed categorisation of participants into underweight (<18.5), normal (18.5 - <25), overweight $(25-<30)$ and ofe $\geq 30$ ) groups. Waist, hip, neck and head circumference were measured usinadical tape measure (Birch, Heidelberg West, Australia), and three skinfold meastrements were taken at the right triceps using a Harpenden Skinfold Caliper (Baty International, West Sussex, UK). An automated BP machine (model 5200-103Z, Welch Allyn, Auckland, New Zealand) was used to measure BP. Two separatemeasurements were taken for systolic and diastolic BP, and the average was receded. If there was a greater difference of $10 \mathrm{mmHg}$ for systolic BP or 5 $\mathrm{mmHg}$ for diastolic BP, a third measurement was taken. The closest BP measurements were then averaged.

Questionnaires

All quj onnaires were professionally translated into the language of the participant. Trained staff then administered the questionnaires with the aid of an interpreter. To 
reduce fatigue, regular breaks were offered throughout the course of the interview. A total of nine questionnaires were completed by each participant, allowing data to be collected in the context of medical, behavioural, lifestyle, psychosocial and genetic risk factors for poor diabetes management. These included a general questionnaire, physical activity questionnaire (DASH2), perceived barriers to diabêtess management (developed in-house), the Summary of Diabetes-Care Activites (ADSCA), diabetes quality of life (ADDQoL), EQ-5D, IIlness Perception Questimaire for diabetes (IPQ-R), the Hospital Anxiety and Depression Scale (HADS and the Self-Efficacy for Diabetes scale (REFS) [5].

U

Retrieyal of vision and refraction data

At the completion of the testing protocol, additional vision and refraction data were collected for each participant from their RVEEH file. Intraocular pressure (Goldmann Applantion or Tonopen in $\mathrm{mmHg}$ ) and any missing socio-demographic details or medicarnistory data, such as GP contact details, medication use and diagnosis and treatneftf eye diseases, were extracted. Participants' files were checked by the inter ensure that all examination information and questionnaires were completed before data entry.

\section{Phenotype definitions}

Good glvagemic control was defined as $\mathrm{HbAlc}<7 \%$ and good BP control as systolic $\leq 130$ and diastolic $\leq 80 \mathrm{mmHg}$. Good diabetes control was defined as having both 1 (6A1c $<7 \%$, and BP systolic and diastolic values of $\leq 130 \mathrm{mmHg}$ and $\leq 80$ $\mathrm{mmHa}$ respectively. DR severity was categorized using the Early Treatment of Diabetinopathy Study levels: no DR, level 10 to 15; mild non-proliferative DR, level 20, moderate non-proliferative, level 31 to 43; severe non-proliferative DR, leveles 0 60; and proliferative DR, level 61-80. Stereoscopic fundus photographs allowed the grading of diabetic macula edema (DME), which was confirmed by OCT. 
Visible retinal thickening or hard exudates in the posterior pole was a positive indicator for the presence of DME. This was further classified into mild (retinal thickening or hard exudate distant from the macula), moderate (retinal thickening or hard exudate approaching the centre of the macula) and clinically significant macular oedema (retinal thickening within a circle of $1 \mathrm{~mm}$ at the centre of the macula). Ungradaliste fundus photographs due to poor image quality or opacity in the media were excluded from the analysis.

Statistiearanalysis

Norma ity the continuous variables was examined using boxplots, KolmogorovSmirngy Shapiro-Wilks tests. Clinical and socio-demographic characteristics of the stuay participants were summarized using mean and standard deviation (SD) for normally ditstributed variables, median and interquartile range (IQR) for nonnormantributed and count variables, and proportions for categorical variables. The chrisquare statistical test was used to analyze differences in proportions betwe ngoups. Comparisons of mean and median values were conducted using an indenant samples t-test or two-sample Wilcoxon rank-sum (Mann-Whitney) test. A two-taind $p$-value $<0.05$ was considered statistically significant.

\section{RESUETS \\ Screengand recruitment}

Out of the 1608 diabetes patients flagged as non-English speaking, 151 (9.4\%) agreed to rarticipate, 39 (2.4\%) were deemed ineligible, 97 (6.0\%) failed to attend their serefuled RVEEH appointment, and 1321 (82.2\%) were missed by recruiters in clinic witurno follow up.

Clinical Attendance 
Of the 151 patients who agreed to participate, 15 (9.93\%) patients did not attend their scheduled appointment, leaving a final cohort of 136 individuals. Although recruitment was an ongoing process, due to a change in administration staff and lack of availability of interpreters at the RVEEH, there were periods (up to 3-6 months) where no recruitment took place.

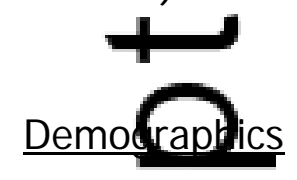

A to 136 participants, 74 males (54.4\%) and 62 females (45.6\%) aged between and 88 years (mean age $=72.2$, standard deviation $[S D]=8.6$ ) were recruited a)d examined in the NEDMP (Table 1). Of these, 111 (82\%) were older than 65 grs and there was no significant age difference for gender $(p=0.726)$. Patients in the NEDMP were significantly older compared to those in the EDMP (mean ade $=64.6, \mathrm{SD}=11.5, \mathrm{p}<0.001$ ). There was a significantly lower proportion of males NEDMP (54.4\%) compared to the EDMP (65.5\%), $p=0.015$. Educaturnal attainment was significantly higher in the EDMP, with only $13.8 \%$ who reportala education level of primary school or below and almost one-third having mor than-14 years education (29.1\%), compared to $43.4 \%$ and $12.5 \%$ in the NEDMP 2 pectively, $\mathrm{p}<0.001$. More participants (28.8\%) in the EDMP reported a higher income bracket $(\geq \$ 30,000)$, compared to those in the NEDMP $(19.8 \%$, $\mathrm{p}<0.0 \mathrm{Q1})$.

\section{Comparion between NEDMP and EDMP}

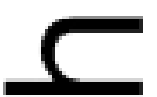

Clinical characteristics and biochemical parameters

In the 83.7\% Tirthe EDMP, $p<0.001$ (Table 1). The median duration of diabetes for partina its in the NEDMP and EDMP was similar, namely 17 years compared to 14 years, $p=0: 087$. The median $\mathrm{HbA}_{1 c}$ for participants in the NEDMP (7.3\%; IQR $=1.3$ ) 
was somewhat lower than that of the $\operatorname{EDMP}(7.5 \%$; IQR $=1.7)$ although the difference was not significant $(p=0.06)$. There was no significant difference in mean SBP in patients from the NEDMP (mean $=143.1 \mathrm{mmHg}$; $\mathrm{SD}=20.6$ ), compared to those in the EDMP (mean=139.6mmHg; $S D=18.8, p=0.140$ ). Similarly, no significant differences were found for DBP in the NEDMP and EDMP: $76.7 \mathrm{mmHg}(\mathrm{SD}=11.3)$ and $76.2 \mathrm{mmHg}(\mathrm{SD}=9.0)$, respectively, $\mathrm{p}=0.468$. Fasting plasma glucose was $7.4 \mathrm{mn} / \mathrm{L} / \mathrm{LQR}=3.0)$ in the NEDMP and $7.7 \mathrm{mmol} / \mathrm{L}(\mathrm{IQR}=3.6)$ in the DMP, $\mathrm{p}=0.087-$ Fable 1 ).

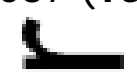

Diabet (CR tinopathy and Diabetic Macular Edema In the NFAMP, $62.7 \%$ (74/118) of patients had DR, with one-third (39/118=33.1\%) having severe NPDR (15/39) or PDR (24/39) (Table 1). Similarly, almost two-thirds of those in the EDMP had DR (364/594=61.3\%), and approximately one-third $(174 / 59-29.3 \%)$ had severe NPDR $(28 / 174=16.1 \%)$ or PDR $(146 / 174=83.9 \%)$. A greaterproportion of patients in the EDMP were found to have at least mild DME (176/55 $1.77 \%$ ) compared to $23.48 \%$ in the NEDMP (27/115), however the diffe

\section{Diabetes Control}

Similar to the EDMP, we found that a higher proportion of patients in the NEDMP with $\mathrm{DP}$ - 7 poor glycemic control (80.8\%. 42/55) compared to those with no DR $(50.0 \% / 34), p=0.003$. There was no significant difference in poor systolic and diasto CBP control between those with and without DR in the NEDMP, 22.9\% (8/34) and $28.4 \%$ (19/55), respectively, $p=0.550$. There were also no significant differes in poor DM control in those with and without DR in the NEDMP, 35.7\% (10/34) and $32.0 \%$ (16/55), respectively $p=0.740$. Overall, for each definition of poor rol (HbAlc alone, BP alone and $\mathrm{HbAlc}$ and BP combined), we found no 
significant difference between the NEDMP and the DMP, $p=0.411, p=0.469, p=$ 0.337 .

\section{DISCUSSION}

We rejort, for the first time, the clinical characteristics and diabetes control in a sample of on-English speaking adults attending tertiary ophthalmic clinics in Melbowastralia. Findings from this study were compared to that of the English speakifromP [5]. We found that the proportion of individuals with type 2 diabetes in the UEDMP (93.4\%) was significantly higher compared to that in the EDMP $(83.7 \%)<0.001$. However, the level of poor diabetes control (HbA1c $\geq 7 \%)$ and the prevalence of non-proliferative DR and vision threatening DR was similar between $t b$ NEDMP and EDMP studies. It is worth noting that the sample size in the NEDMP substantially less than that in the EDMP, 136 versus 613 adults, and thereforeresults must be considered with caution. Our results suggest that the curren diapetes healthcare services, diabetes eye management and awareness progere equally effective in both English and non-English speaking Australians.

There are no comparable studies in Australia on diabetes control and DR severity in non-English speaking Australians. However, our findings concur with that recently reportedin a Canadian based study that assessed the effect of language barriers on the ris acute and chronic complications of diabetes [11]. Although they reported differe ices in socio-demographic risks factors, such as older age and less educational attainment in those with language barriers, they found no differences in the rates diabetes complications. The similarity in the rates of diabetes related complicatrons between English and non-English speaking individuals can be expleine by; 1) non-English speaking adults readily access primary care services; 2) improved communication with family members and hospital based interpreter 
services; 3) translated educational materials and the increase in community support groups [12-14].

We found that more than two-thirds of patients with DR had poor diabetes control in both the EDMP and NEDMP. Similar findings from our work also reflect these unsatistactory levels of diabetes control in English speaking adults [15, 16]. The reason forthese findings may be explained by several factors, such as low adher recommended diabetes related medical examinations by the populativereduced accessibility to specialised senvices, poor understanding on the requir meints to achieve optimal diabetes control and lifestyle risk factors. It is clear that botthipdividual and system barriers need to be addressed to improve diabetes controland prevent the progression of major diabetes related complications, such as sight-threa ening DR.

There were some unique challenges we faced in the NEDMP that need to be highlid fited Firstly, our limited resources only allowed for the recruitment of nonEnglichaking residents who spoke one of five foreign languages. To obtain a wider representation of other languages spoken amongst non-English speaking Australians would require much greater resources (interpreter and translation servic s), funding and time. Secondly, in order to maximise face-to-face recruitpant, it is imperative to employ interpreters specifically for the study, as inhouse meepital interpreters typically have limited research time. Thirdly, greater testing time was required, as it typically involved a three-way communication between the clinical examiner, the interpreter and the participant. Overall, studies into noglish speaking Australians must be well resourced, with adequate funding, manpower and full-time interpreter services. 
The NEDMP has several strengths, including the use of a comprehensive eye testing protocol, collection of behavioural parameters and the assessment of the severity of DR. Although this is a novel study, the methodological issues cannot be ignored. These include a relatively small clinical sample size, only approaching patients who required interpreters covering five languages, and the missed opportunities of recrutiment due to logistical constraints, such as lack of resources (time and funds) and in hou se interpreters. In addition, a comparison between those who were recruitend non-respondents could not be made due methodological and time Furthermore, it is important to consider that differences in the sample size b. twe nn the EDMP and NEDMP, may explain the differences observed in DR severity gotween both studies. These limitations must be considered when interpreting the results included in this paper.

In sumany, we have examined a sample of non-English speaking patients recruited from teruary eye clinics at the RVEEH and found that diabetes control and DR severi 10 pe similar to those recruited in our main English speaking DMP study [5]. Furthandies using a larger sample of non-English speaking Australian residents are requind to determine whether accessibility to diabetes related healthcare services and awareness programs are equally targeted to those with language barriens.

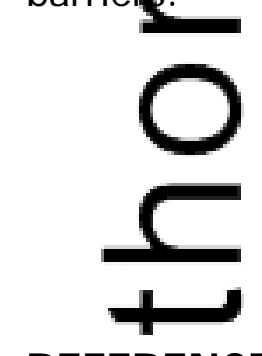
REFERENCES
1. 7 gliano, D.J., et al., Lifetime risk and projected population prevalence of diabetes. Diabetologia, 2008. 51(12): p. 2179-86. 
2. Dunstan, D.W., et al., The rising prevalence of diabetes and impaired glucose tolerance: the Australian Diabetes, Obesity and Lifestyle Study. Diabetes Care, 2002. 25(5): p. 829-34.

3. Yau, J.W., et al., Global prevalence and major risk factors of diabetic retinopathy. Diabetes Care, 2012. 35(3): p. 556-64.

4. IVIrtchell, P., et al., Guidelines for the Management of Diabetic Retinopathy, 2008. National Health and Medical Research Council: Australia.

5. - enoureux, E.L., et al., Methodology and early findings of the Diabetes Hanragement Project: a cohort study investigating the barriers to optimal (diab)tes care in diabetic patients with and without diabetic retinopathy. Clin Eyperiment Ophthalmol, 2012. 40(1): p. 73-82.

6. King, B.R., et al., A diabetes awareness campaign prevents diabetic keto acidosis in children at their initial presentation with type 1 diabetes. Pediatr Diabetes, 2012. 13(8): p. 647-51.

7. Tisu, W.C., et al., Identification of linguistic barriers to diabetes knowledge (ad) glycemic control in Chinese Americans with diabetes. Diabetes Care, 2005. 29(2): p. 415-6.

8.

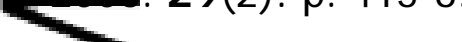

Daster, L.M., et al., Glycemic control in English-vs Spanish-speaking Hispanic patients with type 2 diabetes mellitus. Arch Intern Med, 2001. 161(1): p. 7782.

9. 7heng, Y., et al., Prevalence and risk factors of diabetic retinopathy in migtant Indians in an urbanized society in Asia: the Singapore Indian eye study. Ophthalmology, 2012. 119(10): p. 2119-24.

10. Brooke, P. and R. Bullock, Validation of a 6 item cognitive impairment test a view to primary care usage. Int J Geriatr Psychiatry, 1999. 14(11): p. T50-40.

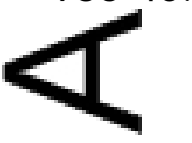


11. Okrainec, K., et al., Impact of language barriers on complications and mortality among immigrants with diabetes: a population-based cohort study. Diabetes Care, 2015. 38(2): p. 189-96.

12. Wang, F., et al., Migration and diabetes in British Columbia and Quebec: prevalence and health service utilization. Can J Public Health, 2012. 103(1): tp. 59-64.

13. Creatore, M.I., et al., Diabetes screening among immigrants: a population- urban cohort study. Diabetes Care, 2012. 35(4): p. 754-61.

14. Lemandez, A., et al., Language barriers, physician-patient language Congordance, and glycemic control among insured Latinos with diabetes: the pirigetes Study of Northern California (DISTANCE). J Gen Intern Med, 2011. 26(2): p. 170-6.

15. Wong, N., et al., Blood pressure control and awareness among patients with dietes and hypertension attending a tertiary ophthalmic clinic. Diabet Med, 2009. 26(1): p. 34-9.

16. Wardg, S., et al., Lack of knowledge of glycosylated hemoglobin in patients diabetic retinopathy. Diabetes Res Clin Pract, 2008. 81(1): p. el5-7.

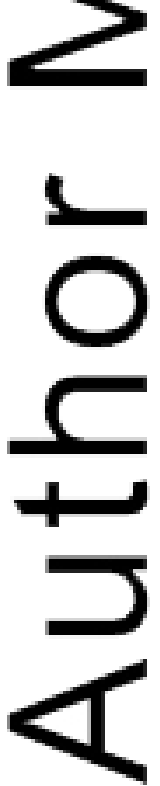




\section{TABLE}

Table 1: Participants' characteristics in the English DMP $(n=609 *)$ and Non-English DMP $(n=136)$

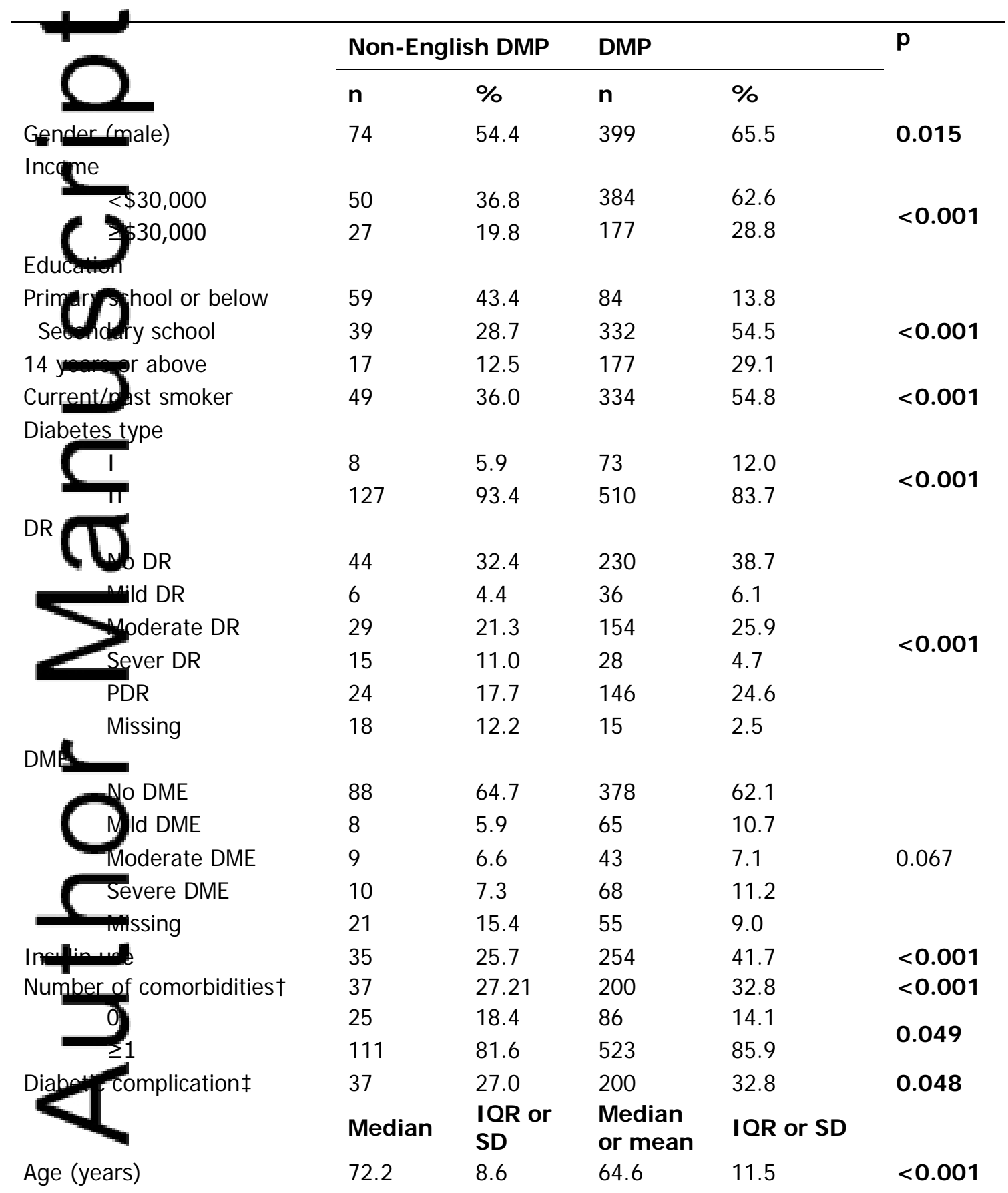




\begin{tabular}{llllll}
$\begin{array}{l}\text { Systolic blood pressure, } \\
\text { mmHg }\end{array}$ & 143.1 & 20.6 & 140.0 & 19.1 & 0.140 \\
$\begin{array}{l}\text { Diastolic blood pressure, } \\
\text { mmHg }\end{array}$ & 76.7 & 11.3 & 76.2 & 9.0 & 0.468 \\
$\begin{array}{l}\text { Duration of diabetes } \\
\text { (years) } \Delta\end{array}$ & 17.0 & 14.0 & 14 & 14.1 & 0.087 \\
$\begin{array}{l}\text { BMl (kg/m²) } \\
\text { Fasting plasma glucose }\end{array}$ & 29.1 & 6.2 & 30.8 & 6.2 & 0.001 \\
(mmol/L) $\Delta$ & 7.4 & 3.0 & 7.7 & 3.6 & $\mathbf{0 . 0 8 7}$ \\
Haemoglobin A1c (\%) $\Delta$ & 7.3 & 1.3 & 7.5 & 1.7 & $\mathbf{0 . 0 5 8}$ \\
Trig (ceringes (mmol/L) $\Delta$ & 1.2 & 0.7 & 1.5 & 1.1 & $<0.001$ \\
\hline
\end{tabular}

$\mathrm{BMI}=$ Rody Mass Index; DMP=Diabetes Management Project

$\Delta$ : as,median (IQR)

† Includeo. hypertension, heart attack/angina, irregular heartbeat, stroke, high cholesfell asthma, anaemia, migraine, arthritis, osteoporosis.

¥Includesw-nephropathy, peripheral vascular disease, neuropathy.

*Four patrents excluded from the analysis as they were found to not have diabetes

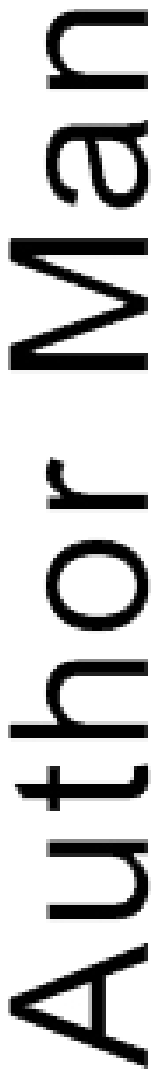

This article is protected by copyright. All rights reserved. 


\section{University Library}

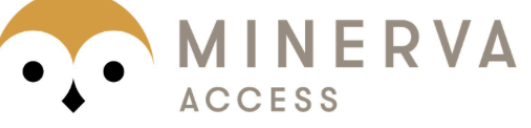

A gateway to Melbourne's research publications

Minerva Access is the Institutional Repository of The University of Melbourne

Author/s:

Dirani, M;Dang, TM;Xie, J;Gnanasekaran, S;Nicolaou, T;Rees, G;Fenwick, E;Lamoureux, EL

Title:

Study methodology and diabetes control in patients from the non-English diabetes management project (NEDMP)

Date:

2017-03-01

\section{Citation:}

Dirani, M., Dang, T. M., Xie, J., Gnanasekaran, S., Nicolaou, T., Rees, G., Fenwick, E. \& Lamoureux, E. L. (2017). Study methodology and diabetes control in patients from the non-English diabetes management project (NEDMP). CLINICAL AND EXPERIMENTAL OPHTHALMOLOGY, 45 (2), pp.160-167. https://doi.org/10.1111/ceo.12807.

Persistent Link:

http://hdl.handle.net/11343/291628 